Dirk Langemann

\title{
Eignen sich kooperative Spiele als Modelle für gesellschaftliche Interaktion?
}

\section{Kommentar zum Beitrag „Die Evolution und der Zusammenbruch von Kooperation: Wie Einzelne das Gemeinwohl gefährden können“von Arne Traulsen}

Kooperative Spiele bzw. Gemeinschaftsgüterspiele konstruieren ein Spannungsfeld zwischen individuellen Bedürfnissen und Interessen einer Gruppe. Gleichzeitig haben die einzelnen Spieler über das Verhalten der Mitspieler und damit über die Auswirkung ihrer individuell wählbaren Handlungsoptionen keine Gewissheit. Vor dem Hintergrund dieser Unsicherheit versuchen sie deshalb solche Handlungen rational auszuwählen, die sie dem Spielziel mit größtmöglicher Wahrscheinlichkeit näherbringen.

Kooperative Spiele enthalten reduzierte Modelle für das Handeln Einzelner in einer z. B. durch Umweltveränderungen bedrohten Gemeinschaft. Dieser Kommentar diskutiert die Frage, ob und in welchem Sinne die Eigenschaften der Modelle Aussagen über gesellschaftliche Vorgänge erlauben und Richtungen realer Entscheidungen implizieren.

\section{Spiele}

Die vorgestellten kooperativen Spiele sind so konstruiert, dass eine Auswahl Einzelner Einschränkungen im Sinne eines klar umrissenen Ziels in Kauf nehmen muss, um Risiken von allen Mitgliedern der Gruppe abzuwenden. Die Regeln der Spiele sind derart gestaltet, dass diejenigen, die zum Erfolg der Gruppe beitragen und die im Sinne der evolutionären Spieltheorie Kooperatoren genannt werden, sowohl im Erfolgsfall als auch im Misserfolgsfall der Gruppe individuell schlechter gestellt sind als diejenigen, die nicht kooperieren. Für den Einzelnen ist es also rational sinnvoll, nicht $\mathrm{zu}$ kooperieren. Interessant ist nun, dass die mehrfache Wiederholung eines solchen Spiels einen sozialen Lernprozess in Gang setzen kann, der trotzdem in einer Kooperation dieser einzelnen, rational handelnden Individuen mündet.

Bevor wir Eigenschaften und Implikationen kooperativer Spiele diskutieren, tragen wir die Besonderheiten der durch diese und andere Spiele geschaffenen Denkumgebung zusammen.

Erstens definieren Spiele genau ein Ziel, und das Spiel ist als Spiel nur dann sinnvoll, wenn das Ziel von den Mitspielern unhinterfragt übernommen wird. Deshalb ist jede Entscheidung und jede Handlung, welche die Mitspieler dem Ziel näherbringt, 
rational sinnvoll, und jede Entscheidung mit einer entgegengesetzten Auswirkung nicht. Insbesondere wenn das Ziel in der Anhäufung einer Messgröße besteht, ist es also sowohl objektiv als auch subjektiv rational sinnvoll, die Messgröße zu mehren.

Zweitens definieren Spiele eine Anzahl von klaren Handlungsoptionen, und diese Anzahl ist endlich und meist sogar sehr klein. Bspw. sehen kooperative Spiele für jeden Einzelnen genau die beiden Optionen vor, zu kooperieren oder nicht zu kooperieren.

Nebenbei bemerkt ist der Begriff der Kooperation in Gemeinschaftsgüterspielen von anderen Kooperationsbegriffen abzugrenzen. Bspw. sieht Adam Smith in der gesellschaftlichen Arbeitsteilung eine Form der Kooperation. Marktwirtschaftliche Wirtschaftstheorien enthalten die Aussage, dass das Streben nach Eigennutz auch dem Gemeinwohl dient. In einer solchen Denkumgebung handelt der Einzelne rational sinnvoll, indem er seinen individuellen Vorteil verfolgt und damit gleichzeitig die Gruppeninteressen voranbringt. Im Gegensatz dazu beinhaltet Kooperation in der evolutionären Spieltheorie die Mitwirkung am Erfolg der Gruppe unter ausdrücklichem Verzicht auf individuelle Vorteile.

Es stellt sich die Frage, was diese Form der Kooperation außerhalb der sehr eingeschränkten Welt der Spiele bedeutet.

\section{Modelle}

Gemeinschaftsgüterspiele werden als Modelle für das Handeln Einzelner in einer Gruppe angesehen, die einem kollektiven Risiko ausgesetzt ist. Bspw. kann eine drohende Umweltkatastrophe ein kollektives Risiko sein, dessen Auswirkungen abgemildert wird, wenn genügend viele Einzelne in Schutzmaßnahmen investieren.

Eine eingeschränkte Variante dieses Szenarios ist ein kooperatives Spiel. Wenn es nur die beiden Optionen gibt, dass Einzelne genau umrissene Schutzmaßnahmen ergreifen oder nicht ergreifen, und wenn wirklich eine bestimmte Anzahl von Einzelnen ausreicht, um die Katastrophe abzuwenden, liegt exakt die oben beschriebene Situation vor. Diejenigen, die kooperieren, profitieren von der ausbleibenden Katastrophe, haben aber individuell investiert. Diejenigen, die nicht kooperieren, profitieren ebenfalls, ohne aber investiert zu haben. Im Misserfolgsfall, also wenn die drohende Katastrophe für alle eintritt, haben die Kooperatoren vorher investiert und die NichtKooperatoren nicht.

Die aufgezählten Gemeinsamkeiten werden allerdings erst durch die idealisierenden Einschränkungen bestimmend. In einer realistischen Situation gibt es in der Regel mehr als zwei Handlungsoptionen. Außerdem ist es im Allgemeinen unbekannt, welche und wie viele Schutzmaßnahmen eine nahende Katastrophe wirksam abwenden.

Ganz allgemein beschreiben Modelle Ausschnitte der Wirklichkeit in idealisierter Form. Rudimentäre Gütekriterien von Modellen sind die Reproduktion von Be- 
obachtungen sowie die Gültigkeit von Vorhersagen. Die Modellbildung greift, soweit möglich, auf bestehende Begriffe und Theorien zurück. Aber je weniger als gesichert angesehene Theorien existieren, desto weniger Anknüpfungspunkte an bestehende Modelle gibt es.

Mit Blick auf das Handeln von Individuen in unserer Gesellschaft entsteht die Frage, ob der gleichzeitige Rückgriff auf die Begriffe und Denkansätze der evolutionären Spieltheorie und der Volkswirtschaftslehre, insbesondere auf den Homo oeconomicus mit seiner rein wirtschaftlichen Rationalität eine vertretbare Idealisierung tatsächlicher Beweggründe von Individuen ist.

Das Konzept des Homo oeconomicus idealisiert vielfältige Interessenlagen auf die Anhäufung der Messgröße Geld und definiert damit ein klar umrissenes Ziel. Ein echter Homo oeconomicus müsste aus allen Handlungsoptionen immer diejenige wählen, die nach Einbeziehung und Abwägung aller Aspekte den größtmöglichen finanziellen Nutzen für ihn selbst verspricht. Dabei ist die Einbeziehung aller Aspekte wichtig, denn auch der ideale Homo oeconomicus würde seine individuelle Gesundheit nicht gegen jeden Geldbetrag eintauschen. Vielmehr würde er, falls so ein Tausch für ihn anstände, den erwarteten Einschränkungen und gesundheitlichen Risiken einen Preis zuweisen und seinen Gesamtgewinn optimieren.

Die Vorstellung, selbst ein Homo oeconomicus zu sein, scheint den meisten Menschen unbehaglich. Sie verweisen dann auf ihre Individualität, ihr Mitgefühl oder auf ihre bewusste Entscheidung für bestimmte Handlungsoptionen unter Berücksichtigung von weit mehr Kriterien als nur ihrem ökonomischen Vorteil.

\section{Logik oder - was ist rational sinnvoll?}

Spiele eignen sich wegen des klar definierten Spielziels dazu, über den Begriff der Logik nachzudenken. Ein Spieler handelt rational sinnvoll oder logisch konsistent, wenn er seine Handlungen so wählt, dass er sich dem Spielziel nähert. Berücksichtigen wir, dass nicht alle Spieler nicht offengelegte Spielsituationen, wie z. B. die Spielkartenverteilung, gleich einschätzen und auch bei Spielen mit vollständiger Information, wie z. B. beim Schach, nicht in gleicher Intensität und Genauigkeit über die Auswahl ihrer Spielzüge nachdenken, so sollten wir eher davon sprechen, dass ein Spieler subjektiv logisch konsistent denkt und subjektiv rational sinnvoll handelt.

Diese subjektive Rationalität schließt Irrtümer ein, denn innerhalb der irrtümlich wahrgenommenen Umgebung handelt der Spieler aus seiner Sicht rational sinnvoll. Auch der strafrechtliche Irrtumsbegriff fußt darauf, dass die betroffenen Menschen innerhalb eines nachvollziehbaren Irrtums rational handeln. Etwa die Behauptung, jemand hätte sich als Kraftfahrzeugführer in dem Irrtum befunden, dass er Kapitän eines Segelschiffes sei und deshalb in Vertretung Hagens von Tronje den Kaplan über Bord werfen musste, bevor er sich wieder dem Straßenverkehr zuwenden konnten, macht aus seinem rechtlichen Problem schnell ein psychiatrisches. 
Kurz gesprochen, kann niemand tatsächlich unlogisch denken, und der Versuch dies zu tun, beweist es eindrucksvoll. Als Gedankenexperiment überzeuge man eine dunkelhaarige Frau von der Schlussfolgerung, dass alle Frauen blond seien, weil Scarlett Johansson blond ist. Menschen halten einen elementaren logischen Widerspruch wie den, dass eine bestimmte Aussage zugleich wahr und nicht wahr ist, nicht aus. In jeder komplizierter verpackten widersprüchlichen Aussage steckt bei genauerer Analyse ein solch elementarer Widerspruch, vgl. der sokratische Elenchos in den platonischen Dialogen.

\section{Altruismus und Gerechtigkeit}

Aus der Behauptung, Menschen würden subjektiv rational sinnvoll handeln, folgt nicht, dass alle Menschen uniform sind und in vergleichbaren Situationen dasselbe tun würden. Außerhalb von Spielen gibt es eine Vielzahl von teilweise konkurrierenden Zielen, eine große Auswahl an Handlungsoptionen, Mischformen von Entscheidungen, und es gibt eine große Variabilität bei der Einschätzung von Situationen.

Insbesondere folgen Menschen nicht den Maximen des Homo oeconomicus. Entgegen diesem Konzept geben Menschen Bettlern Almosen, werfen Geld in den Kollektenbeutel, zahlen die $\mathrm{CO}_{2}$-Abgabe bei Flugtickets und geben selbst dann Trinkgeld, wenn die Bedienung nicht lächelt und wenn sie nie wieder in dieses Lokal gehen wollen. Ein aktuelles Beispiel ist der Kauf von Bioprodukten oder von gerecht gehandelten Waren, wofür es eine Vielzahl von Motiven gibt, aber bestimmt nicht den pekuniären Vorteil.

Einige wenige Menschen richten ihr gesamtes Leben konsequent gemäß einem nicht ökonomisch motivierten Ziel wie der Krankenpflege, der Unterstützung Benachteiligter, dem Klimaschutz, dem Wohl von Mitgeschöpfen, der Rettung der Welt in irgendeinem Sinne oder auch ihrer eigenen inneren Balance aus.

Angesichts der Vielfalt möglicher Ziele ist es nicht immer subjektiv rational sinnvoll, als Homo oeconomicus zu handeln, auch wenn es für den idealisierten Homo oeconomicus immer rational sinnvoll ist, sein Handeln rein ökonomisch auszurichten.

Das sog. Ultimatumspiel, welches in der jüngeren Vergangenheit in medizinischen und behavioristischen Versuchen populär geworden ist, hat die Diskussion um das Konzept des Homo oeconomicus erneut eingefärbt, denn es erscheint als wissenschaftlicher Nachweis der Existenz von altruistischem und nicht gewinnorientiertem Verhalten bei modernen Menschen.

Im Ultimatumspiel wird einem von zwei Spielern ein Gewinn in der Größenordnung von zwanzig Euro zugesagt. Allerdings muss dieser Spieler dem anderen einen Anteil seines vermeintlichen Gewinns anbieten, den der andere Spieler annehmen oder ablehnen kann. Nur wenn das Angebot angenommen wird, dürfen beide Mitspieler ihre Anteile behalten. Sonst bekommen sie nichts. Typischerweise ergeben Experimente, dass Angebote zwischen 30 und 40\% der Gewinnsumme akzeptiert wer- 
den. Kleinere Angebote bringen den zweiten Spieler dazu, das Angebot abzulehnen und selbst ebenfalls leer auszugehen. Man könnte auch sagen, dass es dem zweiten Spieler fünf Euro wert ist, dem ersten Spieler einen Gewinn von fünfzehn Euro zu verderben.

Man kann argumentieren, dass ein Homo oeconomicus als zweiter Spieler jeden noch so kleinen Betrag akzeptieren muss, weil ihn auch der kleine Betrag besser stellt als eine Ablehnung aus Gerechtigkeitsempfinden. Man kann ebenfalls argumentieren, dass ein Homo oeconomicus als erster Spieler einen hohen Betrag anbieten muss, weil der Rest besser als nichts ist. Schließlich ist die Idee nicht abwegig, dass das Szenario des Ultimatumspiels die beiden Spieler nur scheinbar unterscheidet und dass deshalb eine genau hälftige Aufteilung sinnvoll ist.

Da keine dieser Argumentationen durch die Experimente bestätigt wird, wird das Ultimatumspiel nicht nur als Nachweis altruistischen und empathischen Verhaltens angesehen, sondern die angebotenen Summen werden auch als Maß für die Intensität des empathischen Verhaltens verwendet.

Bei genauerer Betrachtung des Ultimatumspiels fällt einerseits auf, dass das nächstliegende Spielziel, möglichst viel Geld mitzunehmen, einen Nutzen außerhalb des Spiels hat. Das Spielziel besteht nicht allein darin, möglichst viel oder wenigstens mehr als die anderen von einer abstrakten Größe zu gewinnen, sondern auch im umsetzbaren Nutzen außerhalb des Spiels. Andererseits gibt es, wie eben dargelegt, nicht genau eine objektiv rationale Strategie für die Höhe des Angebots.

Der stärkste Kritikpunkt an der Interpretation dieser experimentellen Ergebnisse bezieht sich jedoch auf die Höhe der Beträge. Die verwendeten Beträge liegen nur unwesentlich über den Bagatellbeträgen, die in Mitteleuropa als Almosen, Kollekte oder Trinkgeld üblich sind. Die meisten Probanden entscheiden beim nächsten Kneipenbesuch oder Wochenendeinkauf über deutlich höhere Summen. Die möglichen Gewinne sind Bagatellen. Sie zu haben oder nicht zu haben, fühlt sich auch für einen pekuniär orientierten realen Menschen fast gleich an.

Könnte man das Ultimatumspiel um Beträge spielen, die außerhalb der Spielumgebung echte Relevanz haben, würden sich die obigen Prozentangaben vermutlich nicht bestätigen. Der zweite Spieler stände bspw. vor der Entscheidung zwischen einem luxuriösen Urlaub und der Summe null oder aber vor der Entscheidung, ein Jahr lang nicht neben seinem Studium arbeiten zu müssen, oder dies auszuschlagen, weil der erste Mitspieler ein ungerechtes Angebot gemacht hat. Spätestens, wenn die Gewinnsumme ein sonst nicht erfüllbares Bedürfnis oder gar Erfordernis befriedigen kann, wird die Überlegung, welchen Anteil der andere Spieler einstreicht, hinter dem Verhalten zurücktreten, das auch ein Homo oeconomicus rational sinnvoll fände.

Je einschneidender die Beträge für die Betroffenen sind, umso weniger Denkansätze gibt es, in denen ein nicht ökonomisches Verhalten subjektiv rational sinnvoll ist, zumal auch viele nichtmaterielle Güter zumindest gegen viel Geld tauschbar sind. 


\section{Fazit}

Das Szenario einer drohenden Katastrophe, zu deren Abwendung viele einzelne einen erheblichen Beitrag leisten müssen, ist nach diesen Überlegungen in seinem Kern durch Gemeinschaftsgüterspiele in wichtigen Punkten gut abgebildet, d. h. kooperative Spiele sind brauchbare Modelle, um das Zusammenspiel wesentlicher Mechanismen der gesellschaftlichen Interaktion zu beschreiben. Die Umwelt wird durch das Gemeinschaftsgut vertreten und der über Bagatellen hinausgehende benötigte Beitrag der Einzelnen motiviert die Beschreibung der allermeisten Individuen als ökonomisch rational handelnden Homo oeconomicus. Selbst wenn jeder Einzelne neben den ökonomischen Aspekten noch andere Ziele verfolgt, handelt die Gruppe der Einzelnen im Ergebnis annähernd so, als wäre jeder Einzelne ein Homo oeconomicus.

Die evolutionäre Spieltheorie zeigt uns, dass die Kooperation der Individuen sehr brüchig ist und sich nur unter sehr eingeschränkten Bedingungen aus den Eigeninteressen der Individuen entwickelt.

Somit lernen wir aus den Untersuchungen der evolutionären Spieltheorie, dass sich die Hoffnung, die Individuen würden aus altruistischen oder empathischen Motiven zur Abwehr einer drohenden Katastrophe kooperieren, auf sehr speziell konstruierte Fälle beschränken sollte. Die pure Einsicht der Einzelnen ist angesichts der individuellen Nachteile der Kooperatoren und der gleichzeitig erlebten Vorteile der Nicht-Kooperatoren - zumindest vor Eintritt der drohenden Katastrophe - keine Grundlage, auf der rational sinnvolle Strategien gegen den Klimawandel aufbauen können. Erschwerend kommt hinzu, dass der Klimawandel nach bisherigem Kenntnisstand keine zu bestimmten Zeitpunkten drohende Katastrophe ist, die durch einmalige Einsätze genügend vieler Einzelner zu diesen Zeitpunkten abgewehrt werden könnte, sondern ein schleichender Prozess ist, dessen genaue Verlangsamung kaum ermittelbar und erst recht nicht erfahrbar ist. Ein daraufhin angepasstes kooperatives Spiel würde die Handlungsoption der Nicht-Kooperation noch vorteilhafter erscheinen lassen.

Vielmehr erscheint es aus Sicht der Gemeinschaft rational sinnvoll, die Spielregeln, also die Rahmenbedingungen gesellschaftlicher Interaktion -ähnlich der marktwirtschaftlichen Arbeitsteilung im Sinne von Adam Smith - so zu verändern, dass das individuelle Streben nach Eigennutz auch auf diesem Gebiet in Gemeinwohl mündet. 